\title{
Prospects of monoclonal antibodies in COVID-19 treatment: a systematic review.
}

\author{
Emeka Ogiji ${ }^{1}$, Casimir Ofor ${ }^{1}$, Uchenna Ezenkwa $^{1}$, Kingsley Ukwaja ${ }^{2}$, Charles Maduba ${ }^{2}$, \\ Obumneme Ezeanosike ${ }^{2}$, Samuel Ghasi ${ }^{3}$, Anthony Mbah ${ }^{3}$, Chigozie Uneke ${ }^{1}$, and Elvis Shu ${ }^{3}$ \\ ${ }^{1}$ Ebonyi State University \\ ${ }^{2}$ Alex Ekwueme Federal University Teaching Hospital Abakaliki \\ ${ }^{3}$ University of Nigeria - Enugu Campus
}

July 1, 2020

\begin{abstract}
We reviewed the types of monoclonal antibodies being repurposed for COVID-19 therapeutics, the clinical outcomes and adverse effects so as to provide evidence the bedside physicians, the health policy-makers and the general public could employ in the COVID-19 management protocol. This systematic review was conducted following the guidelines of Preferred Reporting Items for Systematic Reviews and Meta-Analyses. The Joanna Briggs Institute's critical appraisal checklists for evaluation of the quality of studies were employed to assess the quality of the different types of primary studies included in the review. Our search strategy identified 396 potentially relevant articles which decreased to 322 after duplicates were removed. 281 articles were screened out due to lack of relevance. The full text of the remaining 41 relevant papers were retrieved for full text evaluation after which only 19 studies from eight countries met our eligibility criteria and were included in the review. Majority (42\%) of the studies emanated from Italy. Also, $94.7 \%$ of the studies used tocilizumab. A total of 698 patients were included in all the studies with a male/female ratio of 1.94:1. 78.9\% of the studies stated patients' co-morbidities which include hypertension (80\%), diabetes mellitus (73.3\%), cardiovascular disease (53.3\%) and obesity (26.7\%). $75.9 \%$ of the patients recovered. Adverse effects reported included viral myocarditis, bacteraemia, candidaemia and invasive aspergillosis. Monoclonal antibodies, especially tocilizumab and eculizumab hold some promise in the treatment of the disease but controlled clinical trials using them as monotherapy are needed to further evaluate this finding.
\end{abstract}

\section{Hosted file}

Prospects of monoclonal antibodies in COVID 19 treatment A systematic review. Final draft.docx available at https://authorea.com/users/338549/articles/465049-prospects-of-monoclonalantibodies-in-covid-19-treatment-a-systematic-review 